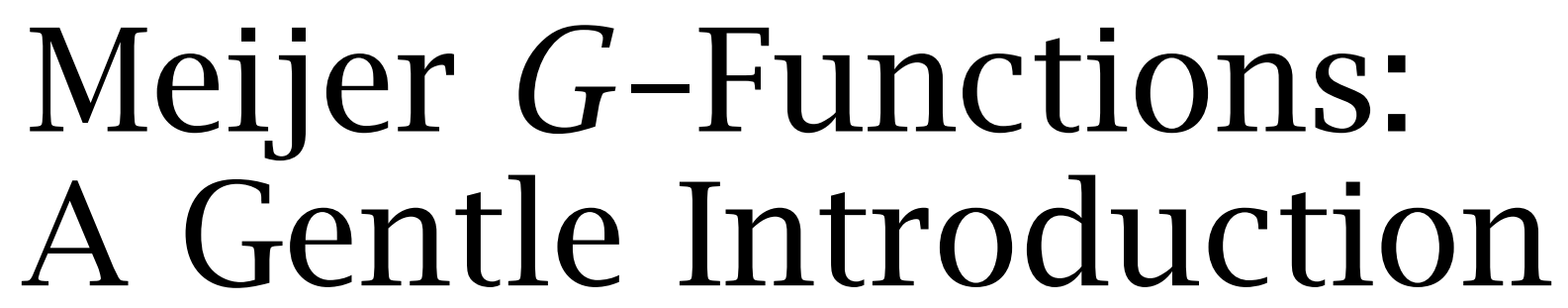

Richard Beals and Jacek Szmigielski

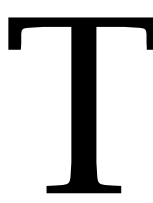

he Meijer $G$-functions are a remarkable family $G$ of functions of one variable, each of them determined by finitely many indices. Although each such function is a linear combination of certain special functions of standard type, they seem not to be well known in the mathematical community generally. Indeed they are not even mentioned in most books on special functions, e.g., [1], [18]. Even the new comprehensive treatise [15] devotes a scant 2 of its $900+$ pages to them. (The situation is different in some of the literature oriented more toward applications, e.g., the extensive coverage in [6] and [9].)

The present authors were ignorant of all but the name of the $G$-functions until the second author found them relevant to his research [3]. As we became acquainted with them, we became convinced that they deserved a wider audience. Some reasons for this conviction are the following:

- The $G$-functions play a crucial role in a certain useful mathematical enterprise.

- When looked at conceptually, they are both natural and attractive.

- Most special functions, and many products of special functions, are $G$-functions or are expressible as products of $G$-functions with elementary functions. There are seventy-five such formulas in [4, sec. 5.6]; see also [6, sec. 6.2], [9, chap. 2], and [20]. Examples are the exponential function, Bessel functions, and products of Bessel functions (the notation will be explained below):

Richard Beals is emeritus professor of mathematics at Yale University. His email address is richard . bea1 s@ya 1e . edu.

Jacek Szmigielski is professor of mathematics at the University of Saskatchewan. His email address is szmigi e1@math. usask.ca.

DOI: http://dx.doi.org/10.1090/notimanid1016

$$
\begin{gathered}
e^{x}=G_{01}^{10}\left({ }_{1} \mid-x\right), \\
J_{v}(2 x)=x^{-v} G_{02}^{10}\left(\begin{array}{ll}
v & 0
\end{array} \mid x^{2}\right),
\end{gathered}
$$

$J_{2 \mu}(x) J_{2 v}(x)=$

$$
\frac{1}{\sqrt{\pi}} G_{24}^{12}\left(\begin{array}{cccc}
\mu+v & v-\mu & \frac{1}{2} & 0 \\
\mu-v & -\mu-v \mid x^{2}
\end{array}\right) .
$$

- The family $G$ of $G$-functions has remarkable closure properties: it is closed under the reflections $x \rightarrow-x$ and $x \rightarrow 1 / x$, multiplication by powers, differentiation, integration, the Laplace transform, the Euler transform, and the multiplicative convolution. Thus, if $G, G_{1}$, and $G_{2}$ belong to $G$ and the various transforms and the multiplicative convolution $G_{1} * G_{2}$ exist, then the following also belong to $G$ :

(1) $G(-x), \quad G(1 / x), \quad x^{a} G(x), \quad G^{\prime}(x)$;

(2) $\int_{c}^{x} G(y) d y$ (for some choice of $c$ );

(3) $\mathcal{L} G(x) \equiv \int_{0}^{\infty} e^{-x y} G(y) d y$;

(4) $\mathcal{E}_{a, b} G(x) \equiv \int_{0}^{1} t^{a-1}(1-t)^{b-1} G(t y) d t$;

(5) $\left[G_{1} * G_{2}\right](x) \equiv \int_{0}^{\infty} G_{1}\left(\frac{x}{y}\right) G_{2}(y) \frac{d y}{y}$.

- The family $G$ is minimal with respect to these properties. For example, the only nonzero multiple of $e^{x}$ that belongs to $G$ is $e^{x}$ itself.

Closure under convolution, (5), is of particular importance for the mathematical enterprise alluded to above. It lies at the heart of the most comprehensive tables of integrals in print [16] and online, as well as the Mathematica integrator; see [19], [6], and [8]. 
In our view, the key to a conceptual understanding of a $G$-function is the differential equation that it satisfies: the generalized hypergeometric equation. We begin by noting what special property singles out precisely these equations among general linear homogeneous ODEs.

\section{The Generalized Hypergeometric Equation}

It is convenient here to replace the operator $d / d x$ with the scale-invariant operator $D=x d / d x$, which is diagonalized by powers of $x$ :

$$
D\left[x^{s}\right]=s x^{s} .
$$

The general homogeneous linear ODE

$$
\begin{aligned}
a_{N}(x) \frac{d^{n}(u)}{d x^{n}}(x) & +a_{N-1}(x) \frac{d^{N-1}(u)}{d x^{N-1}}(x) \\
& +\cdots+a_{0}(x) u(x)=0
\end{aligned}
$$

can, after multiplication by $x^{N}$, be rewritten in the form

$$
\begin{aligned}
b_{N}(x) D^{N} u(x) & +b_{N-1}(x) D^{N-1} u(x) \\
& +\cdots+b_{0}(x) u(x)=0 .
\end{aligned}
$$

Suppose that the coefficients are analytic near $x=0$ and consider the standard power series method: determine the coefficients of a formal power series solution $\sum_{n=0}^{\infty} u_{n} x^{n}$ by expanding (7) and collecting the coefficients of like powers of $x$. Carrying this out by hand can be quite tedious. For example, what are the coefficients $u_{5}$ and $u_{10}$ in the series expansion of the solution of

$$
u^{\prime \prime}(x)+e^{x} u(x)=0,
$$

given that $u_{0}=1, u_{1}=0$ ? This might lead one to ask the following question:

When do the linear equations for the coefficients $\left\{u_{n}\right\}$ in the series expansion reduce to a two-term recursion of the form $c_{n} u_{n}=d_{n} u_{n-1}$ ?

It is not difficult to show that the necessary and sufficient condition on the coefficients $b_{n}$ is that each has the form $\left(\alpha_{n} x+\beta_{n}\right) x^{k}$ for some fixed $k$. It follows that (7) can be reduced to the form

$$
Q(D) u(x)-\alpha x P(D) u(x)=0,
$$

where $Q$ and $P$ are monic polynomials. In view of (6), the recursion for the coefficients of a formal series solution is

$$
Q(n) u_{n}=\alpha P(n-1) u_{n-1} .
$$

If $\alpha=0$, this trivializes: e.g., if the $b_{j}$ are distinct, any solution is a linear combination of powers $x^{1-b_{j}}$. If $\alpha \neq 0$, we may take advantage of the scale invariance of the operator $D$ to take $\alpha x$ as the independent variable and reduce to the case $\alpha=1$.

Finally, the solution is trivial unless $Q(0)=0$. Thus, excluding trivial cases and up to normalization, the answer to the question above is that (7) must be a generalized hypergeometric equation (GHGE), first version:

(9)

$$
\left[D \prod_{j=1}^{q-1}\left(D+b_{j}-1\right)-x \prod_{j=1}^{p}\left(D+a_{j}\right)\right] u(x)=0 .
$$

\section{Generalized Hypergeometric Functions}

For equation (9), the recursion (8) is

(10) $n \prod_{j=1}^{q-1}\left(b_{j}+n-1\right) u_{n}=\prod_{j=1}^{p}\left(a_{j}+n-1\right) u_{n-1}$.

(Here and subsequently we shall assume, usually without explicit statement, various conditions, such as the condition that no $b_{j}-1$ be a negative integer.) The formal power series solution with constant term 1 is

$$
\sum_{n=0}^{\infty} \frac{\left(a_{1}\right)_{n}\left(a_{2}\right)_{n} \cdots\left(a_{p}\right)_{n}}{\left(b_{1}\right)_{n}\left(b_{2}\right)_{n} \cdots\left(b_{q-1}\right)_{n} n !} x^{n},
$$

where the extended factorial $(a)_{n}$ is defined by

$$
\begin{gathered}
a_{0}=1, \\
a_{n}=a(a+1) \cdots(a+n-1)=\frac{\Gamma(n+a)}{\Gamma(a)}, n \geq 1 .
\end{gathered}
$$

The series (11) diverges for all $x \neq 0$ if $p>q$, has radius of convergence 1 if $p=q$, and converges everywhere if $p<q$.

For $p \leq q$ the function defined by the series is the generalized hypergeometric function usually denoted

$$
{ }_{p} F_{q-1}\left(\begin{array}{c}
a_{1}, \ldots, a_{p} \\
b_{1}, \ldots, b_{q-1}
\end{array} \mid x\right) .
$$

Since the equation here has order $q$, there should be an additional $q-1$ linearly independent solutions. We shall come back to this point later.

\section{A More Conceptual Route to a Solution}

We start from a second, slightly generalized, version of (9). The factor $D$ is replaced by $D+b_{q}-1$ :

$$
\left[\prod_{j=1}^{q}\left(D+b_{j}-1\right)-x \prod_{j=1}^{p}\left(D+a_{j}\right)\right] u(x)=0 .
$$

Let us note two features of equation (12). First, $D$ is invariant under $x \rightarrow-x$, so this sign change converts (12) to

(13)

$$
\left[\prod_{j=1}^{q}\left(D+b_{j}-1\right)+x \prod_{j=1}^{p}\left(D+a_{j}\right)\right] u(x)=0 .
$$

Second, under the change of variables $y=1 / x, D$ goes to $-D$. Therefore equation (12) is transformed into one having the same form or else the form (13) (depending on the sign of $(-1)^{q-p}$ ), but with the $q$ parameters $b_{j}$ replaced by the $p$ parameters $1-a_{j}$, and the $p$ parameters $a_{j}$ replaced by the 
$q$ parameters $1-b_{j}$. This allows one to assume always that $p \leq q$.

Since $D$ diagonalizes over powers of $x$, while multiplication by $x$ simply raises the power, we might try to find solutions in the form of (continuous) sums of powers:

$$
u(x)=\frac{1}{2 \pi i} \int_{L} \Phi(s) x^{s} d s,
$$

where $L$ is a suitable closed contour in the complex plane (or the Riemann sphere). We note in passing that integral representations of solutions have a clear advantage over series representations if one wants to determine behavior for large values of $x$ or of the parameters.

Plugging the expression (14) into (12) and assuming that we may differentiate under the integral sign, we want

$$
\begin{aligned}
0= & \frac{1}{2 \pi i} \int_{L} \Phi(s) \\
& \times\left[\prod_{j=1}^{q}\left(b_{j}-1+s\right) x^{s}-\prod_{j=1}^{p}\left(a_{j}+s\right) x^{s+1}\right] d s \\
= & \frac{1}{2 \pi i} \int_{L} \Phi(s) \prod_{j=1}^{q}\left(b_{j}-1+s\right) x^{s} d s \\
& -\frac{1}{2 \pi i} \int_{L+1} \Phi(s-1) \prod_{j=1}^{p}\left(a_{j}-1+s\right) x^{s} d s .
\end{aligned}
$$

If the contour $L$ has the property that $L+1$ can be deformed to $L$ without crossing any singularities of the integrand, then we are led to the continuous version of the recursion (10):

(15) $\Phi(s) \prod_{j=1}^{q}\left(b_{j}-1+s\right)=\Phi(s-1) \prod_{j=1}^{p}\left(a_{j}-1+s\right)$.

We shall refer to this condition on $L$ as the translation condition.

The various issues that arise in carrying out the construction of solutions of (12), by solving (15) and choosing a contour, arise already in the simplest examples.

Example: $q=1, p=0$. We follow the usual convention that the empty product equals 1 . Therefore, with $q=1$ and $p=0$, equation (12) reduces to

$$
(D+b-1) u(x)-x u(x)=0,
$$

and the recursion equation (15) can be written

$$
\frac{\Phi(s)}{\Phi(s-1)}=\frac{1}{b+s-1}=\frac{\Gamma(b+s-1)}{\Gamma(b+s)} .
$$

Therefore we may take $\Phi(s)$ to be the entire function $1 / \Gamma(b+s)$ and set

$$
u(x)=\frac{1}{2 \pi i} \int_{L} \frac{x^{s} d s}{\Gamma(b+s)} .
$$

Now if, as we shall assume, the contour $L$ is closed and does not cross a branch cut of $x^{s}$, then, by
Cauchy's theorem, $u(x)=0$. This leads us to look for another solution of (15).

The product $\varphi(s) \Phi(s)$ is a second solution of (15) if and only if $\varphi(s-1)=\varphi(s)$. In order to remain in the context of gamma functions, we may use Euler's reflection formula,

$$
\Gamma(z) \Gamma(1-z)=\frac{\pi}{\sin \pi z},
$$

and multiply the kernel $1 / \Gamma(b+s)$ in the integral (17) by

(19) $\varphi(s)=\frac{\pi}{\sin \pi(b+s)}=\Gamma(b+s) \Gamma(1-b-s)$, leading to the function

$$
u(x)=\frac{1}{2 \pi i} \int_{L} \Gamma(1-b-s) x^{s} d s .
$$

Note that the factor (19) is antiperiodic rather than periodic with period 1, i.e., $\varphi(s-1)=-\varphi(s)$. This corresponds to changing the sign of $x$ in equation (16). We take as $L$ the loop shown in Figure 1. In fact, the translation condition implies that the poles must lie on one side of $L$, and for a nontrivial result we want $L$ to enclose the poles of the integrand, say in the negative direction. The residue of $\Gamma(1-b-s)$ at $s=1-b+n$ is $(-1)^{n} / n$ !, so (20) is easily calculated:

$$
u(x)=\sum_{n=0}^{\infty}(-1)^{n} \frac{x^{1-b+n}}{n !}=x^{1-b} e^{-x} .
$$

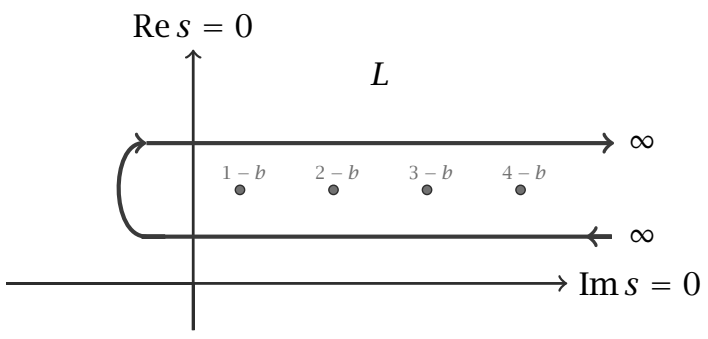

Figure 1. The contour $L$ for (20).

Example: $q=p=1$. Here equation (12) becomes

$$
(D+b-1) u(x)-x(D+a) u(x)=0 .
$$

Equation (15) becomes

$\frac{\Phi(s)}{\Phi(s-1)}=\frac{a+s-1}{b+s-1}=\frac{\Gamma(a+s)}{\Gamma(a+s-1)} \cdot \frac{\Gamma(b+s-1)}{\Gamma(b+s)}$, so one solution is $\Phi(s)=\Gamma(a+s) / \Gamma(b+s)$ :

$$
u(x)=\frac{1}{2 \pi i} \int_{L} \frac{\Gamma(a+s)}{\Gamma(b+s)} x^{s} d s .
$$

This function is meromorphic with poles at $-a$, $-a-1,-a-2, \ldots$ It has at most algebraic growth as $s \rightarrow \infty$ (see the discussion below). Therefore, for $0<|x|<1$ we may take $L$ to be a loop to the right, as in Figure 1. Again, the translation condition requires that the poles lie on one side of this loop, and therefore outside it. Therefore, by Cauchy's theorem, $u(x)=0$ for $0<|x|<1$. 
If $|x|>1$ we may take $L$ to be the loop shown in Figure 2, enclosing the poles.

The residue of the integrand at the pole $s=$ $-a-n$ is

$$
\frac{(-1)^{n}}{n !} \cdot \frac{1}{\Gamma(b-a-n)} \cdot x^{-a-n} .
$$

By (18),

(23)

$$
\begin{aligned}
\frac{1}{\Gamma(b-a-n)} & =\Gamma(1+a-b+n) \frac{(-1)^{n} \sin (b-a)}{\pi} \\
& =(-1)^{n} \frac{\Gamma(1+a-b+n)}{\Gamma(b-a) \Gamma(1+a-b)} \\
& =(-1)^{n} \frac{(1+a-b)_{n}}{\Gamma(b-a)} .
\end{aligned}
$$

Therefore, for $|x|>1$ our solution is

$$
\begin{aligned}
u(x) & =\frac{x^{-a}}{\Gamma(b-a)} \sum_{n=0}^{\infty} \frac{(1+a-b)_{n}}{n !}\left(\frac{1}{x}\right)^{n} \\
& =\frac{x^{-a}}{\Gamma(b-a)}\left(1-\frac{1}{x}\right)^{b-a-1} .
\end{aligned}
$$

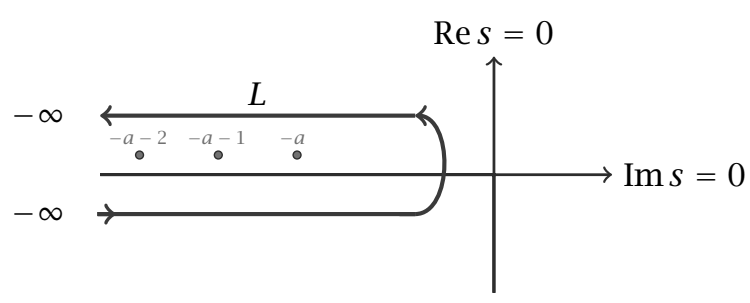

Figure 2. The contour $L$ for (22) when $|x|>1$.

As in the case $p=0$, we may change the integrand, leading, for example, to

$$
u(x)=\frac{1}{2 \pi i} \int_{L} \frac{\Gamma(1-b-s)}{\Gamma(1-a-s)} x^{s} d s .
$$

Again for $0<|x|<1$, we may integrate over a contour that encloses the poles going to the right, as in Figure 1. For $|x|>1$ we choose a contour going to the left, as in Figure 2, but enclosing no poles. A calculation as in (23), (24), together with Cauchy's theorem, gives

$$
u(x)=\left\{\begin{array}{cc}
\frac{x^{1-b}(1-x)^{b-a-1}}{\Gamma(b-a)}, & 0<|x|<1 ; \\
0, & |x|>1 .
\end{array}\right.
$$

An interesting special case is $a=0, b=1$, which gives a step function:

$$
u(x)=H(1-|x|), \quad x \neq 0,
$$

where $H$ is the Heaviside function.

We leave it to the reader to consider two more possibilities, with respective kernels $\Gamma(a+s) \Gamma(1-$ $b-s)$ and $1 /[\Gamma(1-a-s) \Gamma(b+s)]$.

\section{Representation by Generalized Hyper- geometric Functions}

Before proceeding to a discussion of the general case, we look briefly at the second order equation

$$
\begin{aligned}
{\left[\left(D+b_{1}-1\right)\left(D+b_{2}-1\right)\right.} \\
-x(D+a) u(x)] u(x)=0 .
\end{aligned}
$$

One possibility for a solution is

(25)

$u(x)=\frac{1}{2 \pi i} \int_{L} \Gamma(a+s) \Gamma\left(1-b_{1}-s\right) \Gamma\left(1-b_{2}-s\right) x^{s} d s$.

Another way to construct a solution is to look for $u_{1}(x)=x^{1-b_{1}} v_{1}(x)$. The corresponding equation for $v_{1}$ is

$$
\left[D\left(D+b_{2}-b_{1}\right)-x\left(D+a+1-b_{1}\right)\right] v_{1}(x)=0 \text {, }
$$

which has a solution in standard form:

$$
v_{1}(x)={ }_{1} F_{1}\left(\begin{array}{c}
a+1-b_{1} \\
b_{2}+1-b_{1}
\end{array} \mid x\right),
$$

and similarly with $b_{1}$ and $b_{2}$ interchanged. The solution (25) must be a linear combination of these two. The coefficients can be determined by looking at the residues of the integrand at the pole $s=1-b_{1}$ and at the pole $s=1-b_{2}$ : $\Gamma\left(a+1-b_{1}\right) \Gamma\left(b_{1}-b_{2}\right)$ and $\Gamma\left(a+1-b_{2}\right) \Gamma\left(b_{2}-b_{1}\right)$ respectively. The result is

$$
\begin{aligned}
u(x)= & \Gamma\left(a+1-b_{1}\right) \Gamma\left(b_{1}-b_{2}\right) \\
& \times x^{1-b_{1}}{ }_{1} F_{1}\left(\begin{array}{c}
a+1-b_{1} \\
b_{2}+1-b_{1}
\end{array} \mid x\right) \\
& +\Gamma\left(a+1-b_{2}\right) \Gamma\left(b_{2}-b_{1}\right) \\
& \times x^{1-b_{2}}{ }_{1} F_{1}\left(\begin{array}{c}
a+1-b_{2} \\
b_{1}+1-b_{2}
\end{array} \mid x\right) .
\end{aligned}
$$

There is one notable feature of this particular combination of the two standard solutions $v_{1}, v_{2}$. Each of the $v_{j}$ has exponential growth as $x \rightarrow+\infty$, but (27) does not. In fact, the integrand decays exponentially in both directions on vertical lines, so we may deform the contour $L$ into one that consists of a small circle around $s=a$, together with a vertical line $\operatorname{Re} s=c<\operatorname{Re} a$. The leading term can be computed from the residue at $s+a=0$ : (28)

$u(x) \sim \Gamma\left(a+1-b_{1}\right) \Gamma\left(a+1-b_{2}\right) x^{-a}$ as $x \rightarrow+\infty$.

Thus this integral form singles out the unique (up to a multiplicative constant) linear combination of the two standard solutions that has algebraic behavior at $+\infty$.

\section{General Considerations about Kernels and Contours}

For each case of equation (12), the function $\Phi$ that satisfies the recursion equation (15) can and will be taken to be a quotient of products of gamma functions in the form $\Gamma\left(-c_{j}+s\right)$ or $\Gamma\left(d_{j}-s\right)$. 
Therefore the poles of $\Phi$, if any, will consist of finitely many sequences of points:

(a) $c_{j}, c_{j}-1, c_{j}-2, \ldots$ or (b) $d_{j}, d_{j}+1, d_{j}+2, \ldots$.

The translation condition requires that any such sequence must lie on one side of the contour $L$. The contour $L$ is always chosen to separate the sequences (a) of poles that go to the left from the sequences (b) that go to the right. (A technical remark: We do not actually need the translation condition to deduce that these solutions of (15) give rise to solutions of the generalized hypergeometric equation; the condition that $L$ separate the sequences of poles in this way is sufficient.)

We consider three types of contours $L$ :

$L=L_{I}$ : beginning at $-i \infty$, ending at $+i \infty$;

$L=L_{\infty}$ : beginning and ending at $+\infty$, oriented clockwise;

$L=L_{-\infty}$ : beginning and ending at $-\infty$, oriented counterclockwise.

In order to see which contours are available in a given case, we note here some basic facts about asymptotics.

First, Stirling's formula,

$$
\Gamma(z)=\sqrt{\frac{2 \pi}{z}}\left(\frac{z}{e}\right)^{z}\left[1+O\left(\frac{1}{z}\right)\right] \text { as } z \rightarrow \infty,
$$

is valid uniformly in the right half-plane $\operatorname{Re} z \geq 0$. It implies that $\Gamma$ grows faster than exponentially to the right along any horizontal line and decays exponentially in both directions along any vertical line in the right half-plane.

Second, the reflection formula (18), combined with (29), gives the asymptotic behavior in the left half-plane $\operatorname{Re} z \leq 0$ : $\Gamma$ decays faster than exponentially to the left along any horizontal ray other than the negative real axis and decays exponentially in either direction along any vertical line in the left half-plane as well. These facts imply that, for any fixed $a, b$,

$$
\frac{\Gamma(a+s)}{\Gamma(b+s)} \sim s^{a-b} \quad \text { as } s \rightarrow \infty
$$

along any ray that avoids the zeros and poles of the quotient.

\section{Meijer $G$-functions}

Here we consider the general hypergeometric equation (12) but with a difference from the standard notation: the indices $\left\{b_{j}\right\},\left\{a_{j}\right\}$ are replaced by the reflections $\left\{1-b_{j}\right\},\left\{1-a_{j}\right\}$ :

$$
\left[\prod_{j=1}^{q}\left(D-b_{j}\right)-x \prod_{j=1}^{p}\left(D+1-a_{j}\right)\right] u(x)=0 .
$$

We assume again that $p \leq q$. The previous considerations lead us to a solution which, in Meijer's notation, is $G_{p, q}^{0, p}$ :

$$
\begin{aligned}
G_{p, q}^{0, p} & \left(\begin{array}{l}
a_{1}, \ldots, a_{p} \\
b_{1}, \ldots, b_{q}
\end{array} \mid x\right) \\
& =\frac{1}{2 \pi i} \int_{L} \frac{\prod_{j=1}^{p} \Gamma\left(1-a_{j}+s\right)}{\prod_{j=1}^{q} \Gamma\left(1-b_{j}+s\right)} x^{s} d s .
\end{aligned}
$$

Meijer [10] introduced a family of solutions of equation (31), denoted by

$$
\begin{array}{r}
G_{p, q}^{m, n}\left(\begin{array}{l}
a_{1}^{\prime}, \ldots, a_{p}^{\prime} \\
b_{1}^{\prime}, \ldots, b_{q}^{\prime}
\end{array} \mid(-1)^{m+n+p_{X}}\right), \\
0 \leq m \leq q, \quad 0 \leq n \leq p,
\end{array}
$$

where the $\left\{a_{j}^{\prime}\right\}$ and $\left\{b_{j}^{\prime}\right\}$ are permutations of the original indices $\left\{a_{j}\right\}$ and $\left\{b_{j}\right\}$ respectively. The upper index $m$ indicates that the first $m$ factors in the denominator of the integrand have been changed to factors $\Gamma\left(b_{j}-s\right)$ in the numerator and the last $p-n$ factors in the numerator have been changed to factors $\Gamma\left(a_{j}-s\right)$ in the denominator. This results in a total of $m+n$ factors in the numerator.

Taking into account the invariance of the integrand under permutations of the indices $a_{j}$ and $b_{j}$ (separately) in the numerator and also in the denominator, one can obtain $2^{p+q}$ solutions of (31). However they are not necessarily distinct. If $p<q$, then the $2^{p}$ solutions with $m=0$ vanish identically, as in our first solution (17) in the case $q=1, p=0$. If $p=q$, only the solution with $m=n=0$ vanishes identically.

The choice of contours depends first on $p$ and $q$. If $p<q$ (resp. $p>q$ ), the gamma function kernel $\Phi$ has faster than exponential decay to the right (resp. left) on horizontal lines. Thus, for $p<q$, one can take a contour $L=L_{\infty}$ as in Figure 1, and for $p>q$ a contour $L=L_{-\infty}$ as in Figure 2. In either case the contour is chosen to separate the sequences of poles going to $+\infty$ from those going to $-\infty$.

If there are more gamma factors in the numerator than in the denominator, i.e., $m+n>(p+q) / 2$, then $\Phi$ decays exponentially along vertical lines and the contour can be deformed into $L_{I}$ from $-i \infty$ to $+i \infty$. If $m+n=(p+q) / 2$, $\Phi$ has algebraic behavior at $\infty$ by (30), and again the contour can be deformed, but this may require interpreting the integral in the sense of distributions.

When $p=q$, the kernel $\Phi$ itself has algebraic behavior as $s \rightarrow \infty$, just as in the case $p=q=1$ above, so the choice of contour differs according to whether $0<|x|<1$ or $|x|>1$.

A striking advantage of this method of producing solutions is the ease of finding solutions with prescribed behavior as $x \rightarrow 0$ or $x \rightarrow \infty$. Assuming again that $p \leq q$, the standard solutions of the form $x^{b_{j}} p F_{q-1}$ that are $x^{b_{j}}[1+(O(x)]$ as $x \rightarrow 0$ are 
multiples of the $G$-functions with $m=1$. Solutions that are $x^{a_{j}-1}\left[1+O\left(x^{-1}\right]\right.$ as $x \rightarrow \infty$ are multiples of the $G$-functions with $n=1, m+1>(p+q) / 2$.

\section{The Mellin Transform and the Convolution Theorem}

The Mellin transform is the multiplicative version of the Laplace transform. We normalize it as

$$
\mathcal{M} f(s)=\int_{0}^{\infty} f(x) x^{-s} \frac{d x}{x} .
$$

The inverse transform is then

$$
\mathcal{M}^{-1} g(x)=\frac{1}{2 \pi i} \int_{c-i \infty}^{c+i \infty} g(s) x^{s} d s .
$$

This suggests, correctly, that if a $G$-function

$$
G(x)=\frac{1}{2 \pi i} \int_{L} \Phi(s) x^{s} d s
$$

has (in some suitable sense) a Mellin transform, then that transform is the kernel $\Phi$. As an exercise, one can check this with (24): change the variable of integration to $y=1 / x$ to find that the Mellin transform is a beta function and therefore expressible in terms of gamma functions.

It is not difficult to verify that the Mellin transform takes multiplicative convolution to a product: in the notation of (5),

$$
\mathcal{M}\left(G_{1} * G_{2}\right)=\mathcal{M} G_{1} \cdot \mathcal{M} G_{2} .
$$

If $G_{1}$ and $G_{2}$ are $G$-functions with $\mathcal{M} G_{j}=\Phi_{j}$, then the product $\Phi_{1} \Phi_{2}$ is itself a quotient of products of gamma functions. If

$$
G_{j}=G_{p_{j}, q_{j}}^{m_{j}, n_{j}}, \quad j=1,2,
$$

then $G_{1} * G_{2}$ is a $G$-function of type

$$
G_{p_{1}+p_{2}, q_{1}+q_{2}}^{m_{1}+m_{2}, n_{1}+n_{2}}
$$

Some bookkeeping will identify the indices $\left\{a_{j}\right\}$, $\left\{b_{j}\right\}$ for $G$ in terms of the indices $\left\{a_{j, 1}\right\},\left\{b_{j, 1}\right\}$ for $G_{1}$ and $\left\{a_{j, 2}\right\},\left\{b_{j, 2}\right\}$ for $G_{2}$.

This explains the convolution result (5). We leave verification of the closure under (1), (2), (3), and (4) to the reader.

We note in passing that the most commonly used integral transforms, such as the Laplace, Hankel, and fractional integral transforms, as well as the Mellin transform itself, can be viewed as special cases or variants of the general $G$-function transform

$$
T f(x)=\int_{0}^{\infty} G(x t) f(t) d t .
$$

Conversely, the range of the $G$-function transform can be characterized in terms of the special cases just mentioned; see [17].

\section{Discussion and History}

The one bit of motivation that we have found in the literature is the remark in [4] that "the $G$-function provides an interpretation of the symbol ${ }_{p} F_{q}$ when $p>q+1$." Let us start from the equation

$$
\begin{array}{r}
{\left[\prod_{j=1}^{p}\left(D+a_{j}-1\right)-x \prod_{j=1}^{q}\left(D+b_{j}\right)\right] v(x)=0,} \\
p \geq q .
\end{array}
$$

As we noted earlier, under the changes of variables $x \rightarrow \pm 1 / x$ the operator $D$ goes to $-D$. Therefore if we set

$$
u(x)=v\left(\frac{(-1)^{p-q}}{x}\right),
$$

equation (33) is equivalent to equation (31). This explains the departure in (31) from the usual index notation (12).

We cannot help noting that the remark is of limited explanatory value (apart from the question of notation), since ${ }_{p} F_{q-1}$, with $p \leq q$ and a given set of indices, denotes a single solution to (9), not $2^{p+q}$ solutions.

Meijer's original definition in 1936 [10] is the general version of (27): represent the function as a linear combination of the standard basis of solutions of (31). (This definition tacitly rules out the cases with index $m=0$, which give the trivial solution.) Only in [11] did Meijer present the integral formulation above. The 1936 paper translates many formulas for special functions of the form

$$
f(x)=h(x) k(x)
$$

or

$$
f(x)=h_{1}(x) k_{1}(x)+h_{2}(x) k_{2}(x)
$$

into a form like

$$
G(x)=G_{1}(x) G_{2}(x),
$$

and many integral formulas into the convolution form

$$
G(x)=G_{1} * G_{2}(x) .
$$

It appears that Meijer may simply have done so many calculations that he could see exactly what linear combinations would make these formulas work out so neatly. (He may also have been guided by known examples that yield specified asymptotics, as in the example (27) above.) Only later did Meijer establish the integral formulas like (32) that are now taken as the definition [10]. In a lengthy series of papers he explored further relations with classical special functions, relations among the $G$-functions themselves, and questions of asymptotics [12], [13]; see also [5].

The idea to represent solutions of the GHGE as integrals of the kind we have been discussing-a type now known as Mellin-Barnes integrals-goes back to Barnes in 1908 for the Euler-Gauss case $q=p=2$ [2] and to Mellin in 1910 for the general 


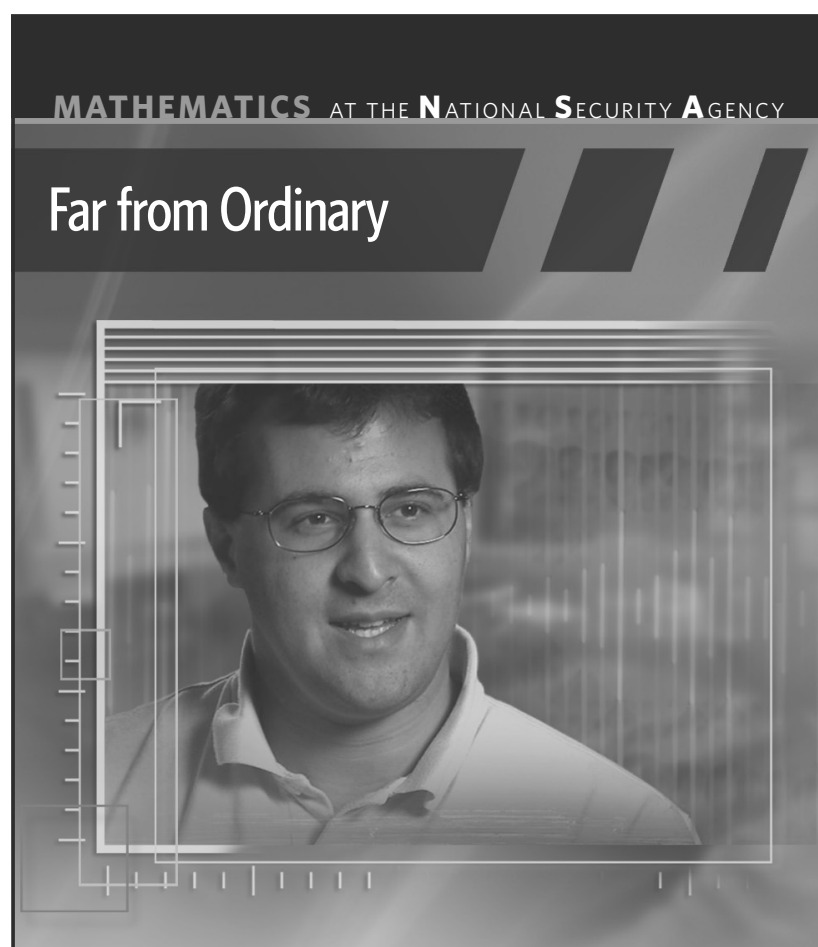

The U.S. is under cyber attack, every minute of every day. That's why cyberspace has become today's new front line. What you know can make a difference at the National Security Agency. Whether it's collecting foreign intelligence or preventing foreign adversaries from accessing U.S. secrets, you can protect the nation by putting your intelligence to work. Explore technology that's years ahead of the private sector. Plus exciting career fields, paid internships, co-op and scholarship opportunities. See how you can be a part of our tradition of excellence and help the nation stay a step ahead of the cyber threat.

\section{KNOWINGMATTERS}

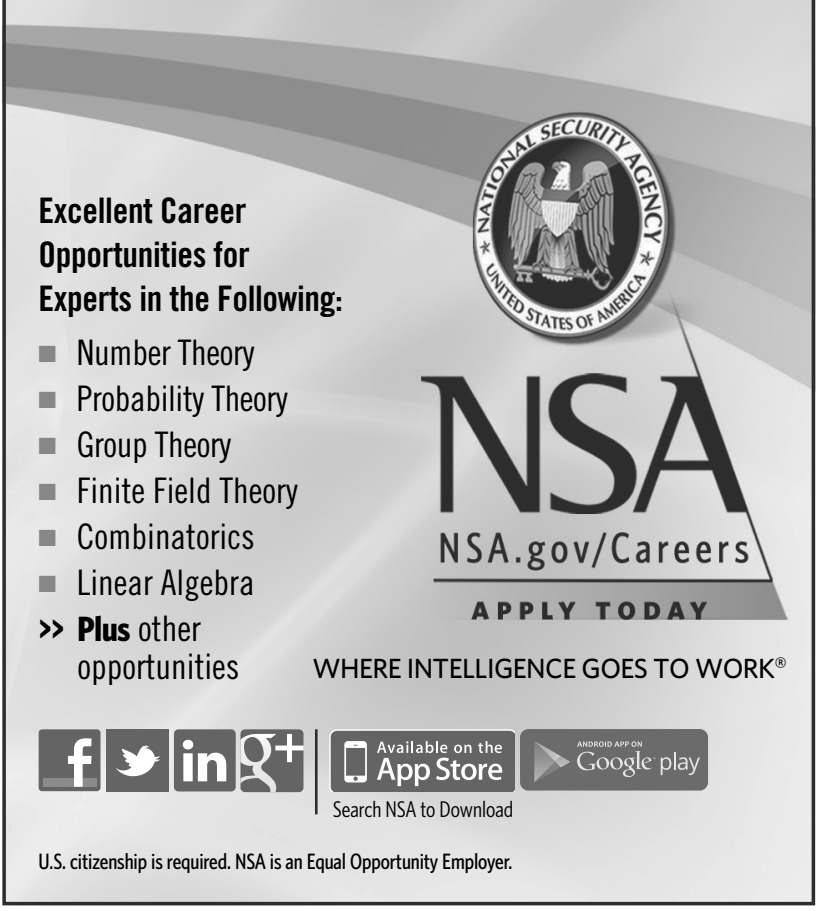

case [14]. Indeed, Mellin has a thorough discussion of the recursion equation (15) and its solutions.

\section{References}

[1] G. E. ANDrews, R. AsKey, and R. Roy, Special Functions, Cambridge Univ. Press, Cambridge, 1999.

[2] E. W. BARNES, A new development of the theory of the hypergeometric function, Proc. London Math. Soc. (2) 6 (1908), 141-177.

[3] M. Bertola, M. Gekhtman, and J. SzmigielSKI, Cauchy-Laguerre two-matrix model and the Meijer-G random point field, 2012, http://arxiv.org/abs/1211.5369[math.PR]

[4] A. ERDÉLYI, W. MAGNUS, F. OBERHETTINGER, and F. Tricomi, Higher Transcendental Functions, Vol. 1, Krieger, Melbourne, FL, 1981.

[5] J. L. FIELDS, The asymptotic expansion of the Meijer $G$-function, Math. Comp. 26 (1972), 757-765.

[6] D. LichtBlAU, Symbolic definite integration, http://1ibrary.wolfram.com/infocenter/ Conferences/5832/.

[7] Y. L. LuKE, The Special Functions and Their Approximations, Vol. 1, Academic Press, New York, 1969.

[8] O. I. MARICHEV, Handbook of Integral Transform of Higher Transcendental Functions, Wiley, New York, 1983.

[9] A. M. MATHAi and R. K. SAXENA, Generalized Hypergeometric Functions with Applications in Statistics and Physical Sciences, Springer, Berlin, 1973.

[10] C. MEIJER, Über Whittakersche bezw. Besselsche Funktionen und deren Produkten, Nieuw Arch. Wisk. 18 (1936), 10-39.

[11] $G_{p, q}^{m, n}(z)$, Nederl. Akad. Wetensch., Proc. Ser. A 44 (1941), 1062-1070.

[12] _ On the G-functions, I-VII, Nederl. Akad. Wetensch., Proc. Ser. A 49 (1946), 344-356, 457-469, 632-641, 765-772, 936-943, 1063-1072, 1165-1175.

[13] , Expansion theorems for the $G$-function, I-XI, Nederl. Akad. Wetensch., Proc. Ser. A 55 (1952), 369379, 483-487; 56 (1953), 43-49, 187-193, 349-357, 57 (1954), 77-82, 83-91, 273-279; 58 (1955), 243-251, 309-314; 59 (1956), 70-82.

[14] HJ. Mellin, Abriss einer einheitlichen Theorie der Gamma-und der hypergeometrischen Funktionen, Math. Ann. 68 (1910), 305-337.

[15] F. OlVER, D. LOZIER, R. BOISVERT, and C. ClARK, NIST Handbook of Mathematical Functions, Cambridge Univ. Press, Cambridge, 2010; NIST Digital Library of Mathematical Functions, http://d7mf.nist.gov,

[16] A. P. PRudnikov, Yu. A. BRYChKOV, and O. I. MARICHEV, Integrals and Series, 5 vols., Gordon and Breach, Newark, NJ, 1986-1992.

[17] P. G. RoONEY, On integral transforms with $G$-function kernels, Proc. Royal Soc. Edinburgh A 93 (1982/83), 265-297.

[18] N. M. Temme, Special Functions, an Introduction to the Classical Functions of Mathematical Physics, Wiley, New York, 1996.

[19] S. WOLFRAM, Festschrift for Oleg Marichev, http:// www.stephenwolfram.com/publications/ recent/specialfunctions/intro.html.

[20] WOLFRAM RESEARCH, MeijerG, http://functions. wolfram.com/HypergeometricFunctions/MeijerG/ 